\title{
A model to investigate the feasibility of FDG as a surrogate marker of hypoxia
}

Kelly, C.J. and Smallbone, K. and Roose, T. and Brady, J.M.

2007

MIMS EPrint: 2009.20

Manchester Institute for Mathematical Sciences

School of Mathematics

The University of Manchester

\footnotetext{
Reports available from: http://eprints.maths.manchester.ac.uk/

And by contacting: The MIMS Secretary

School of Mathematics

The University of Manchester

Manchester, M13 9PL, UK
} 


\title{
A MODEL TO INVESTIGATE THE FEASIBILITY OF FDG AS A SURROGATE MARKER OF HYPOXIA.
}

\author{
Catherine J Kelly ${ }^{1}$, Kieran Smallbone $e^{2}$, Tiina Roose ${ }^{3}$ and Sir Michael Brady ${ }^{1}$ \\ ${ }^{1}$ Wolfson Medical Vision Laboratory, University of Oxford \\ ${ }^{2}$ Manchester Centre for Integrative Systems Biology, University of Manchester \\ ${ }^{3}$ Centre for Mathematical Biology and Oxford Centre for Industrial and Applied Mathematics, \\ University of Oxford
}

\begin{abstract}
Fmiso-PET is a non-invasive modality used for the assessment of tumour hypoxia, and increasingly for planning radiotherapy. However, the availability and contrast properties of Fmiso are not ideal. Recent efforts to compare FDG binding with that of Fmiso, in order to ascertain FDG's potential as a marker of hypoxia, have met with mixed results. The potential reasons for correlated and disparate binding patterns between the two tracers have been postulated, but not formally outlined as yet. We present a model of a key component of the image formation process - tracer pharmacokinetics. This involves a series of coupled PDEs, describing the interplay between concentrations of oxygen, glucose, HIF, Fmiso and FDG. We use this model to assess the general feasibility of FDG as a surrogate marker of hypoxia and find that its utility is dependent on activity of oncogenic pathways.
\end{abstract}

Index Terms-Positron Emission Tomography, Biomedical image processing, Biological Systems Modeling

\section{INTRODUCTION}

One of the current challenges in molecular imaging is the noninvasive imaging of tumour hypoxia. Determining the oxygenation status of a tumour is crucial as hypoxia both affects the efficacy of radiotherapy and is also indicative of a malignant phenotype.

Positron Emission Tomography (PET) offers a non-invasive means of assessing tumour hypoxia. Its utility is dependent on the specificity and sensitivity of the tracer used. The standard hypoxia tracer $[18 \mathrm{~F}]$-fluoromisonidazole (Fmiso), is specifically reduced in hypoxic tissues, becoming covalently bound to intracellular molecules. However, this specificity is compromised by poor distribution kinetics. The lipophillic nature of the compound means that it distributes into all tissues, with typical tumour-to-blood ratios of approximately 1.2 .

Far better distribution characteristics are exhibited by the metabolic tracer, [18F]-fluorodeoxyglucose (FDG), a hydrophillic molecule, whose entry to cells is transporter-mediated. Conveniently, FDG has a direct link to tumour hypoxia, as the transcription factor hypoxia-inducible factor (HIF) controls regulation of genes encoding glycolytic enzymes [1]. Recently, there have been several attempts in the clinic to assess the feasibility of FDG as a surrogate marker for hypoxia [2][3]. These have demonstrated mixed results, with some correlation between Fmiso and FDG reported within cancer types, but less correlation within individual cancers.

Thanks to the Medical Imaging and Signals Inter-disciplinary Research Consortium for funding.
Elevated levels of HIF are found in the nucleus within minutes of cellular exposure to hypoxia. Glycolytic enzymes are upregulated and glycolysis increases approximately 2 and a half fold. As a protective mechanism, oxidative phosphorylation is reduced concomitantly to negligible levels by pyruvate dehydrogenase kinase (PDK), a HIF-mediated enzyme.

The regulation of glycolysis in tumours is complex and extends beyond HIF. The Warburg effect, where cells utilise glycolysis even under aerobic conditions, can be initiated both via and in the absence of HIF by oncogenic pathways such as the Akt pathway [4]. Akt upregulates glycolysis by both stabilising HIF and, independently, activating hexokinase- 2 and glucose transporters.

In this paper, we describe a key component of the imaging process - tracer pharmacokinetics. We present a formal model of both HIF- and oncogenic pathway-mediated glycolysis and use this as a basis to describe FDG and Fmiso kinetics in response to hypoxia. A model to simulate the kinetics of Fmiso has been described previously [5]. In that work, a system of equations described oxygen and Fmiso kinetics. Here, we adapt this work to incorporate FDG binding.

We use our model to (i) investigate the relationship between the microenvironmental oxygenation and tracer binding, and (ii) to compare the different effects of oncogenic pathway activation on FDG binding. Finally, based on the results of these studies, we discuss the feasibility of FDG as a surrogate marker of hypoxia.

\section{MODEL}

A simplified model of HIF-mediated metabolic response to hypoxia is presented in Figure 1. The two main processes mediated by HIF are (i) the upregulation of glycolysis and (ii) the downregulation of oxidative phosphorylation, the former clearly leading to increased FDG avidity. The activation of oncogenic pathways (e.g. Akt) has two potential effects. The first is stabilisation of HIF levels. The second is the HIF-independent upregulation of glycoysis. The latter suggests a potential divergence between hypoxia- and oncogenemediated FDG binding.

The model dynamics are described using the system of nondimensionalised PDEs outlined in Equations 1-5. The main variables are HIF $(H)$, Oxygen $(O)$, Glucose $(G)$, FDG $(F)$ and FMISO $(M) . H$ is produced and retained intracellularly in response to local oxygen concentration. Nutrients $(O$ and $G$ ) and tracers ( $F$ and $M)$ are supplied by the blood and then diffuse through a network of 'cells', being metabolised or bound, depending on local environmental conditions and the oncogenic state of each cell. 


$$
\begin{array}{cccccc}
\frac{\partial H}{\partial t} & = & r_{H}(1-H) & - & d_{H}^{\star} H\left(\frac{O}{k_{H}+O}\right) \\
\frac{\partial O}{\partial t}= & - & d_{O}\left(\frac{O}{k_{O}+O}\right)(1-\gamma H(t-\tau)) & + & D_{O} \nabla^{2} O \\
\frac{\partial G}{\partial t}= & - & d_{G}^{\star}\left(\frac{G}{k_{G}+G}\right)\left(1+k_{A} H\right) & + & D_{G} \nabla^{2} G \\
\frac{\partial F}{\partial t}= & - & d_{G}^{\star}\left(\frac{F}{k_{G}+G}\right)\left(1+k_{A} H\right) & + & D_{G} \nabla^{2} F \\
\frac{\partial M}{\partial t}= & - & d_{M} M\left(\frac{k_{m M}}{k_{m M}+O}\right) & + & D_{M} \nabla^{2} M
\end{array}
$$

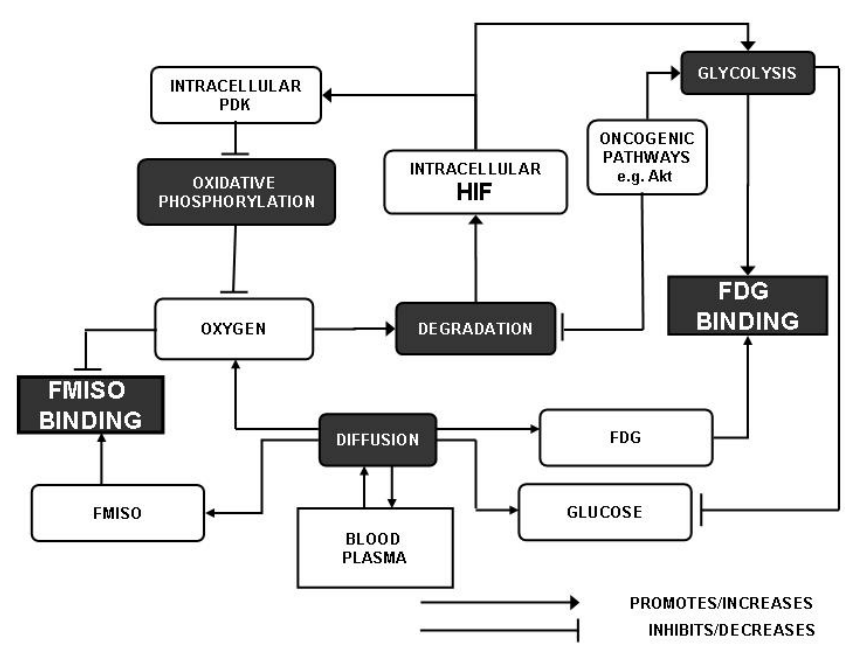

Fig. 1. Proposed model of HIF- and oncogenic pathway-mediated glycolysis and the relationship with FDG and Fmiso binding. Molecular components (e.g. tracers) are shown in white boxes. Regulatory and diffusive processes are shown in black boxes. Oncogenic pathways can affect glycolysis (and thus FDG binding) directly through upregulation of enzymes, or indirectly through HIF stabilisation.

Activation of the oncogenic pathway is represented as a random occurrence. Once activated, two processes are affected:

- Degradation of HIF is inhibited (stabilisation)

- Glycolysis is upregulated (Warburg)

These are represented as a decrease in the maximal rate of HIF degradation $\left(d_{H}^{\star}\right)$ and an increase in the basal rate of glucose consumption $\left(d_{G}^{\star}\right)$. Under normal conditions these are $d_{H}^{n}$ and $d_{G}^{n}$ (normal), and under oncogenic activation these are $d_{H}^{a}$ and $d_{G}^{a}(a \mathrm{kt})$. As little comparative quantitative data yet exists for HIF-dependent and independent Akt-mediated glycolysis, values for $d_{H}^{a}$ and $d_{G}^{a}$ are estimated as multiples of their normal counterparts, i.e. $d_{H}^{a}=\frac{1}{\alpha} d_{H}^{n}$ and $d_{G}^{a}=\beta d_{G}^{n}$. To investigate the relative effects of each pathway on tracer uptake, we compare profiles of FDG and Fmiso for $\alpha=1$, $\beta=1$ (no oncogenic regulation), $\alpha=2, \beta=1$ (HIF stabilisation) and $\alpha=1, \beta=2$ (HIF-independent regulation of glycolysis).
The concentrations presented here are normalized by the maximal plasma or intracellular level of each component, to give relative quantities e.g. $F$ is the concentration of FDG relative to its maximum plasma level. By looking at relative quantities, trends and relationships are often clearer. Equations were scaled to a time scale of 1 minute to best represent changes during a conventional PET scan, and a spatial scale of 1 cell width $(20 \mu \mathrm{m})$. Full parameterisation is available in the appendix (Section 6).

Our model equations were integrated in Matlab using the finite difference method. We model our physical system as the simple onedimensional case in which a single vessel supplies tissue. Boundary conditions are Dirichlet at the vessel and Neumann at the distal end of the grid.

\section{RESULTS}

\subsection{Molecular Profiles}

We initially present profiles of the non-dimensionalised concentrations described above, as a function of increasing distance from a blood vessel, in the absence of oncogenic pathway activation. Profiles for total concentration are shown in Figure 2 (tracers will exist in free and bound states, but imaging will only show the combined concentration). Oxygen and glucose are both depleted as distance increases. Oxygen is almost fully metabolised after only a few cells widths, in agreement with previous calculations [6]. HIF increases with distance, although levels plateau. Both Fmiso and FDG show an increase in total concentration.

Inspection of the temporal profiles of total Fmiso and FDG for normoxic (cell adjacent to blood vessel) and hypoxic (6 cells away from blood vessel) tissue is shown in Figure 3. Contrast between activity in hypoxic and normoxic tissues develops at later time points. Due to the slower diffusive properties of Fmiso, contrast is obtained later than using FDG. Maximum contrast during the scan period is higher for FDG than for Fmiso (1.7 and 1.32 respectively).

\subsection{Effect of Oncogenic Pathways}

Profiles in the previous section are generated under the assumption that increased glycolysis is entirely hypoxia mediated. In this section we present profiles of FDG in the presence of oncogenic pathway activation.

Figure 4 shows the normoxia/hypoxia temporal profile of FDG where $\alpha=2$ and $\beta=1$, i.e. when the oncogenic pathway 


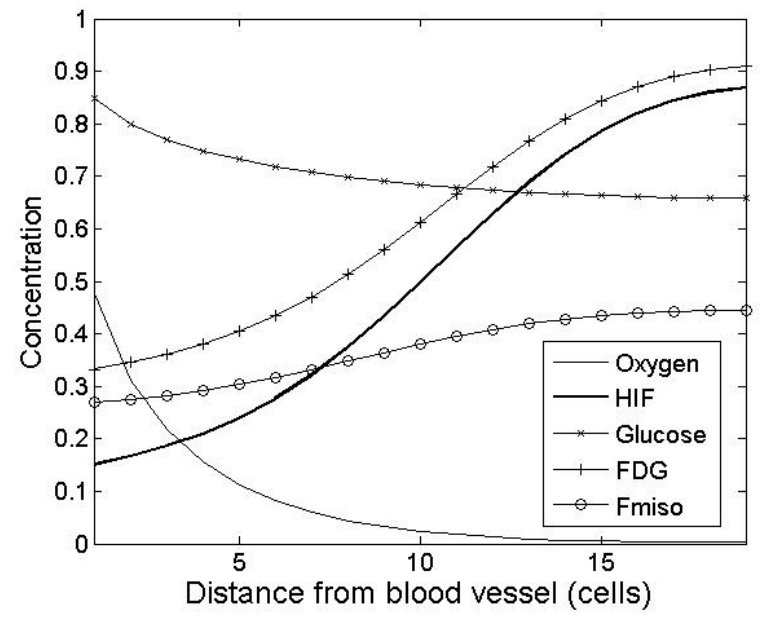

Fig. 2. Concentration profiles with increasing distance from blood vessel

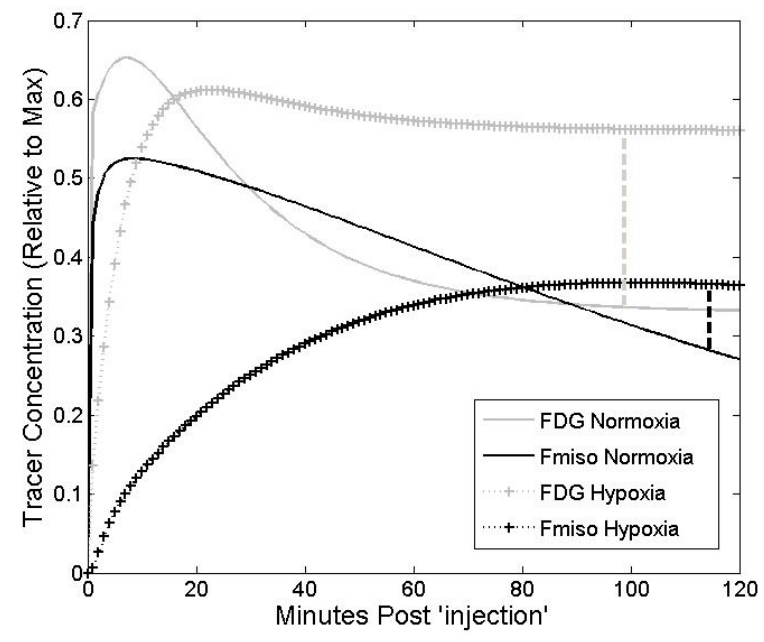

Fig. 3. Temporal Profiles of FDG and Fmiso for normoxic and hypoxic tissue. Dashed lines indicate hypoxia:normoxia contrast. Max contrast Fmiso: 1.32, FDG: 1.7.

acts through HIF stabilisation alone. Figure 5 shows the normoxia/hypoxia temporal profile of FDG where $\alpha=1$ and $\beta=2$, i.e. when the oncogenic pathway acts through HIF-independent upregulation of glycolysis. These figures show that hypoxia:normoxia contrast is reduced in both instances (from 1.7 to 1.6 for HIF-dependent Akt activity, and 1.4 for HIF-independent Akt activity), but that the action of HIF stabilisation, as it is represented in our model, is relatively small compared to the dominant effect of HIF-independent upregulation of glycolysis. Also evident from Figure 5 is that FDG levels in Akt-regulated normoxic tissue could potentially match or even supersede those in HIF-regulated hypoxic tissue, depending on the value of $\beta$.

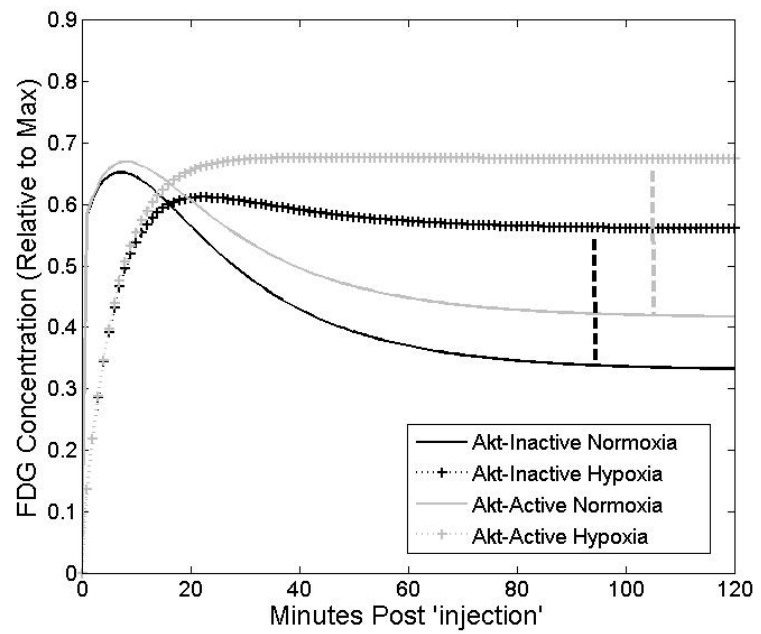

Fig. 4. Temporal Profiles of FDG for normoxic and hypoxic tissue under Akt-mediated HIF stabilisation $(\alpha=2, \beta=1)$, compared to Akt-inactive tissue ( $\alpha=1, \beta=1)$. Dashed lines indicate hypoxia:normoxia contrast. Max contrast Akt-inactive: 1.7, Aktactive: 1.6

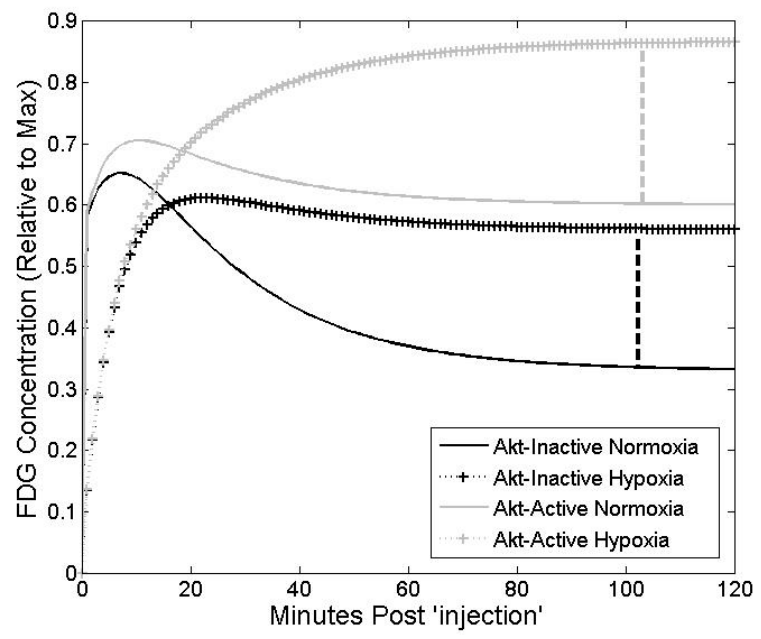

Fig. 5. Temporal Profiles of FDG for normoxic and hypoxic tissue under Akt-mediated upregulation of glycolysis $(\alpha=1, \beta=2)$ compared to Akt-inactive tissue $(\alpha=1, \beta=1)$. Dashed lines indicate hypoxia:normoxia contrast. Max contrast Akt-inactive: 1.7, Akt-active: 1.4.

\section{DISCUSSION AND SUMMARY}

This model highlights several significant differences between what can be inferred, regarding hypoxia, from FDG and Fmiso images. Figure 3 would suggest that in the absence of other glycolytic regulators, FDG would be a sound candidate for a surrogate marker of hypoxia, as proposed previously [7]. Its uptake is increased in the 
presence of hypoxia and less time is required to achieve contrast than when using Fmiso. However, regulation of glycolysis by other factors is an issue. Akt acts via both stabilisation of HIF levels and direct upregulation of glycolytic enzymes, and has been shown to enhance FDG uptake in mice transfected with Akt-active cells [4]. Whilst HIF stabilisation has a minor effect on FDG uptake, Figure 5 demonstrates that HIF-independent regulation of glycolysis (Warburg Effect) could be a key factor in lack of correlation between FDG and Fmiso binding in clinical images. Any non-hypoxia mediated regulation of FDG may potentially lead to a reduction in hypoxia:normoxia contrast. Additionally, as tumours are highly heterogeneous, binding in hypoxic tissue that is not Akt-active could be masked by that in normoxic Akt tissue.

Although FDG is only an independent marker of hypoxia for hypoxia-regulated tumours, this in itself is potentially useful. If FDG and Fmiso are used in conjunction with one another, the pattern of correlation may provide further tumour characterisation. High correlation between tracers may indicate a lack of oncogenic pathway activation and thus a better prognosis. Low correlation, where FDG binds and Fmiso does not, may indicate enhanced oncogenic activity. These are presently just hypotheses and require further investigation.

To summarise, in the absence of alternative regulation, our model would suggest that FDG is a good candidate for a surrogate marker of hypoxia. However, when other regulating factors are considered, it becomes clear that FDG can no longer act as an independent hypoxic marker. The potential exists for using Fmiso and FDG as complementary imaging agents, to provide a more complete tumour characterisation. This complementarity presents a strong argument for image fusion.

\section{ACKNOWLEDGEMENTS}

The authors wish to acknowledge the advice of Professor Adrian Harris and his research group at the Weatherall Institute of Molecular Medicine, University of Oxford. We would also like to thank Dr Mark Gooding of the Nuffield Department of Obstetrics and Gynaecology, University of Oxford, for his assistance in model development.

\section{APPENDIX}

\begin{tabular}{|c|c|c|}
\hline Symbol & Value & Details \\
\hline$r_{H}$ & $6.9 \cdot 10^{-3}$ & Maximum HIF production rate constant \\
\hline$d_{H}^{n}$ & $5.1 \cdot 10^{-2}$ & $\begin{array}{l}\text { Maximum HIF degradation rate con- } \\
\text { stant }\end{array}$ \\
\hline$k_{H}$ & 0.15 & $\begin{array}{l}\text { Oxygen concentration at half maximum } \\
\text { degradation rate }\end{array}$ \\
\hline$d_{O}$ & 2.8 & Oxygen consumption $\mathrm{V}_{\max }$ \\
\hline$k_{O}$ & 0.15 & Oxygen consumption $\mathrm{k}_{\mathrm{m}}$ \\
\hline$\gamma$ & 0.99 & Proportion of reduction \\
\hline$D_{O}$ & $2.2 \cdot 10^{2}$ & Oxygen diffusion coefficient \\
\hline$d_{G}^{n}$ & $9.4 \cdot 10^{-3}$ & $\begin{array}{l}\text { Glucose consumption } V_{\max } \text { (normal } \\
\text { tissue) }\end{array}$ \\
\hline$k_{G}$ & $1 \cdot 10-2$ & Glucose consumption $\mathrm{k}_{\mathrm{m}}$ \\
\hline$k_{A}$ & 1.4 & $\begin{array}{l}\text { Effect of HIF on Glycolysis rate con- } \\
\text { stant }\end{array}$ \\
\hline$D_{G}$ & 84 & Glucose diffusion coefficient \\
\hline$d_{M}$ & 0.11 & Maximal Fmiso binding $\mathrm{V}_{\max }$ \\
\hline$k_{m}$ & $3 \cdot 10-2$ & Fmiso consumption $\mathrm{k}_{\mathrm{m}}$ \\
\hline$D_{M}$ & 8.4 & Fmiso diffusion coefficient \\
\hline$\tau$ & $9.6 \cdot 10^{2}$ & Transcriptional delay \\
\hline
\end{tabular}

\section{REFERENCES}

[1] GL Semenza, PH Roth, HM Fang, and GL Wang, "Transcriptional regulation of genes encoding glycolytic enzymes by hypoxia-inducible factor 1," J Biol Chem, vol. 269, pp. 2375763, 1994.

[2] LM Cher, C Murone, N Lawrentschuk, S Ramdave, A Papenfuss abd A Hannah, GJ O'Keefe, JI Sachinidis, SU Berlangieri, G Fabinyi, and AM Scott AM, "Correlation of hypoxic cell fraction and angiogenesis with glucose metabolic rate in gliomas using $18 \mathrm{f}$-fluoromisonidazole, $18 \mathrm{f}$-fdg pet, and immunohistochemical studies.," J Nucl Med, vol. 47(3), pp. 410-8, 2006.

[3] JG Rajendran, DA Mankoff, and F O' Sullivan, “Hypoxia and glucose metabolism in malignant tumors: evaluation by [18f]fluoromisonidazole and [18f]fluorodeoxyglucose positron emission tomography imaging.," Clin Cancer Res, vol. 10, pp. 2245-52, 2004.

[4] R.L Elstrom, D.E Bauer, M. Buzzai, R. Karnauskas, M.H. Harris, D.R. Plas, H. Zhuang, R.M Cinalli, A. Alavi, C.M. Rudin, and C.B. Thompson, "Akt stimulates aerobic glycolysis in cancer cells,” Cancer Res, vol. 64, pp. 3892-3899, 2004.

[5] CJ Kelly and JM Brady, "A model to simulate tumour oxygenation and dynamic [18f]-fmiso pet data.," Phys. Med. Biol., vol. 51, pp. 5859-73, 2006.

[6] RH Thomlinson and LH Gray, "The histological structure of some human lung cancers and the possible implications for radiotherapy.," Br J Cancer, vol. 9, pp. 539-49., 1955.

[7] A Pugachev, S Ruan, S Carlin, S M Larson, J Campa, C C Ling, and J L Humm, "Dependence of FDG uptake on tumor microenvironment.," Int J Radiat Oncol Biol Phys, vol. 62(2), pp. 545-53, 2005. 